\title{
Effects of functionalization on the electronic and absorption properties of the smaller diamondoids: a computational study
}

\author{
MUHAMMED JENEESH K K and R PADMANABAN* \\ Department of Chemistry, Pondicherry University, R V Nagar, Kalapet, Puducherry 605 014, India \\ E-mail: rpn.che@pondiuni.edu.in
}

MS received 14 April 2018; revised 25 May 2018; accepted 29 May 2018; published online 2 August 2018

\begin{abstract}
Functionalized diamondoids show great potential as building blocks in view of their optoelectronic, catalytic, and therapeutic applications. In this work, we report the effect of functionalization on the electronic and absorption properties of the first two lower diamondoids, adamantane and diamantane, respectively. We performed quantum mechanical calculations within the framework of density functional theory (DFT) and timedependent (TD-DFT) theory for the mono-, di-, and tetra-substituted smaller diamondoids. Bandgap tuning is made through the push (electron-donating) and pull (electron-withdrawing) doping, external substitution at the $\mathrm{C}-\mathrm{H}$ site, which reduces the band gap of diamondoids significantly. The stability of pristine and the functionalized smaller diamondoids is also studied by calculating the ionization potentials and affinity energies. Employing TD-DFT method at the level of PBE1PBE theory, the UV absorption spectra of the functionalized smaller diamondoids were calculated and discussed. While the lowest allowed and most intense transition energies are significantly reduced with the increasing number of pull-type functional groups, the push-type groups slightly increase those energies.
\end{abstract}

Keywords. Functionalized diamondoids; TD-DFT study; absorption spectra; ionization potential.

\section{Introduction}

In recent years, the functionalization of diamondoids has attracted many researchers due to their numerous applications in synthetic chemistry, biomedicine, material science, nanotechnology and beyond. ${ }^{1-6}$ Diamondoids are cage-like, ultra-stable, saturated hydrocarbon molecules that are superimposable on the diamond cubic lattice, and they are also known as nanodiamonds (nanometer-sized, hydrogen-terminated diamond-like molecules). They are chemically and thermally stable due to their exceptional atomic arrangements. To add a few, they have (i) very high melting points as compared with other hydrocarbons, (ii) negative electron affinities, and (iii) high electrical insulating properties as diamond. It is well-known that the basic repetitive unit of diamondoids is a ten-carbon ( $\mathrm{sp}^{3}$ hybridized) tricyclic cage system called adamantane $\left(\mathrm{C}_{10} \mathrm{H}_{16}\right)$, which consists of the cyclohexane rings fused in the chair conformation. The first diamondoid unit (adamantane) was discovered in 1933. It was isolated from the petroleum sample by Landa and Machacek, ${ }^{7}$ and the direct synthesis was done by Schleyer in $1957 .{ }^{8}$ The successive units of diamondoids, with the generic formula $\left[\mathrm{C}_{4 \mathrm{n}+6} \mathrm{H}_{4 \mathrm{n}+12}\right]$, diamantane $(n=2)$, triamantane $(n=3)$, and so on for higher units $(n>3)$ were also isolated from fossil fuels or synthesized in laboratories. ${ }^{9-12}$ Higher diamondoids exhibit increasing structural complexity and a vast number of isomeric forms, and therefore they are very difficult to synthesize, ${ }^{11}$ whereas the lower diamondoids $(n \leq 3)$ exists in only one form. Due to their unique structure and properties, these discoveries have steadily increased the research interests of using diamondoids and its derivatives as readily available precursors, i.e., molecular building blocks for the nanoscience and nanotechnology communities, chemical industry and polymer synthesis. ${ }^{13}$ As for an application to pharmaceutics, amine-derivatives of the adamantane are already in use as anti-viral and anti-Parkinson's agents. ${ }^{14}$

Apart from many synthetic reports, ${ }^{9-12,15}$ there are some experimental and theoretical studies which discuss the physicochemical ${ }^{16}$ and optoelectronic

\footnotetext{
*For correspondence

Electronic supplementary material: The online version of this article (https://doi.org/10.1007/s12039-018-1505-5) contains supplementary material, which is available to authorized users.
} 
properties ${ }^{17-20}$ of the diamondoids and its derivatives. Experimentally, the solubility of the lower diamondoids and their derivatives in various organic solvents has been measured by Chan et al., ${ }^{16}$ for the temperatures range $\sim 273$ to $348 \mathrm{~K}$. In that, they have observed higher solubility in nonpolar solvents than in polar solvents for pure diamondoids, on the other hand, the hydroxyl functionalized diamondoids enhance the solubility in polar solvents within the temperature range, and also observed that the solubility was not size-dependent. Tuning the electronic and optical properties of the smallest diamondoid was achieved by Landt and coworkers ${ }^{20}$ through a single thiol functionalization in the gas phase experiments. Their findings reveal that the optical properties of the diamondoids were strongly affected due to an additional density of states that arises from the $S$ electron lone pair, i.e., optical-gap of adamantane-1-thiol is lowered by $\sim 0.6 \mathrm{eV}$ and UV luminescence is quenched. Their study on the absorption properties ${ }^{20 \mathrm{~b}}$ shows that the absorption in the deep UV regime depends on the functionalization site (apical, medial, and secondary position) and not size-dependent.

From a theoretical point of view, the size effects on the energetic and electronic properties of the diamondoids were predicted by Tomanek and co-workers ${ }^{18}$ through local density approximation (LDA) calculations. The electronic properties such as ionization potentials, affinity energies and HOMO-LUMO gaps of the small diamondoids have been reported elsewhere ${ }^{21}$ using the $a b$ initio methods. Compared to the pure diamondoids, their functionalized forms have many potential applications as a result of tuning the structure and properties. Several functionalizations (interstitial or substitutional doping to the $\mathrm{CH}$ or $\mathrm{CH}_{2}$ site) approaches have been reported in the literature, ${ }^{22-26}$ for e.g., thiolated diamondoids shows high negative electron affinity and low optical gaps, ${ }^{17}$ bandgap tuning by push-pull doping, ${ }^{22-24}$ and very recently, methyl-functionalized diamondoids shown to fluoresce in the gas phase. ${ }^{27}$ In support of interstitial doping, Fyta's research group ${ }^{26}$ contributed significantly to understand the structural characteristics, energetic, and electronic properties of diamondoids and their analogous, such as boron-nitride diamondoids, carbene-functionalized diamondoids, and diamondoidsbased molecular junctions. Their results are based on the quantum mechanical calculations within the density functional theory (DFT) approach. Similar to BN-based diamondoids, ${ }^{26}$ theoretical models of the complete interstitial doping (replacing all $\mathrm{C}$ atoms of diamondoid unit) by semiconductor material, such as $\mathrm{AlSb}, \mathrm{InSb}$, and InAs-based diamondoids were also reported in the literature. ${ }^{28}$ Very recently, Fyta's group have reported the electronic and optical properties of the small diamondoids but for the selected functional groups (push-type, $-\mathrm{NH}_{2}$ and -SH only) of the present study. ${ }^{26 \mathrm{~d}}$

Despite the well-known structures of diamondoids and their derivatives, understanding their absorption and electronic properties is still under intense research. In this work, we present such study by performing the first-principle quantum mechanical calculations using the time-dependent density functional approach. To shed light on the optical properties and excited states influenced by the functionalization, we here systematically investigate the smaller diamondoids and its functionalized forms obtained by mono, di and tetra substitution(s) with the push (electron donors) or pull (electron acceptors) doping. In the following sections, we briefly discuss the computational methodology of our investigation and analyze the results of pure and functionalized diamondoids. Finally, the paper is concluded with the summary of this work.

\section{Computational Methods}

In this work, the first-principles calculations were performed using Gaussian09 software package ${ }^{29}$ within the KohnSham framework of DFT and time-dependent DFT (TD-DFT) for the smaller diamondoids and its derivatives in the gas phase. The hybrid exchange functional of Perdew-BerkeErnzerhof (known as PBE in the literature ${ }^{30}$ ) together with a $6-31+G(d, p)$ basis set was used, unless otherwise stated, in our calculations. At first, the optimized geometry of all the molecules in their ground state was obtained using the DFT method at the level of PBE1PBE theory. It yields the total energies and energy gaps of the functionalized molecules considered for the present study. The energy gap between the frontier molecular orbitals (highest occupied molecular orbital (HOMO) and lowest unoccupied molecular orbital (LUMO)) is often used to measure the optical properties. Subsequently, the frequency calculations were performed which confirm no imaginary frequencies for the selected (pristine and functionalized) diamondoids and ensure that the molecules possess stable structures. Using the ground state (optimized) geometries, the absorption spectra were calculated by considering the maximum of 25 lowest singlet excited states for the selected diamondoids. The main features of the spectra are presented by means of the vertical excitation energies and oscillator strengths corresponding to the lowest allowed and intense transitions. For this purpose, we employed the same level of theory within the TD-DFT formalism, ${ }^{31}$ and the visualization of molecular orbitals and electron densities are done with GaussView5. ${ }^{32}$

In order to study the stability of the functionalized diamondoids, the vertical ionization potentials (IP) and electron affinities (EA) were also evaluated as energy difference in the total energies of the ionic part (either cation or anion) and its relaxed neutral species, given by, 

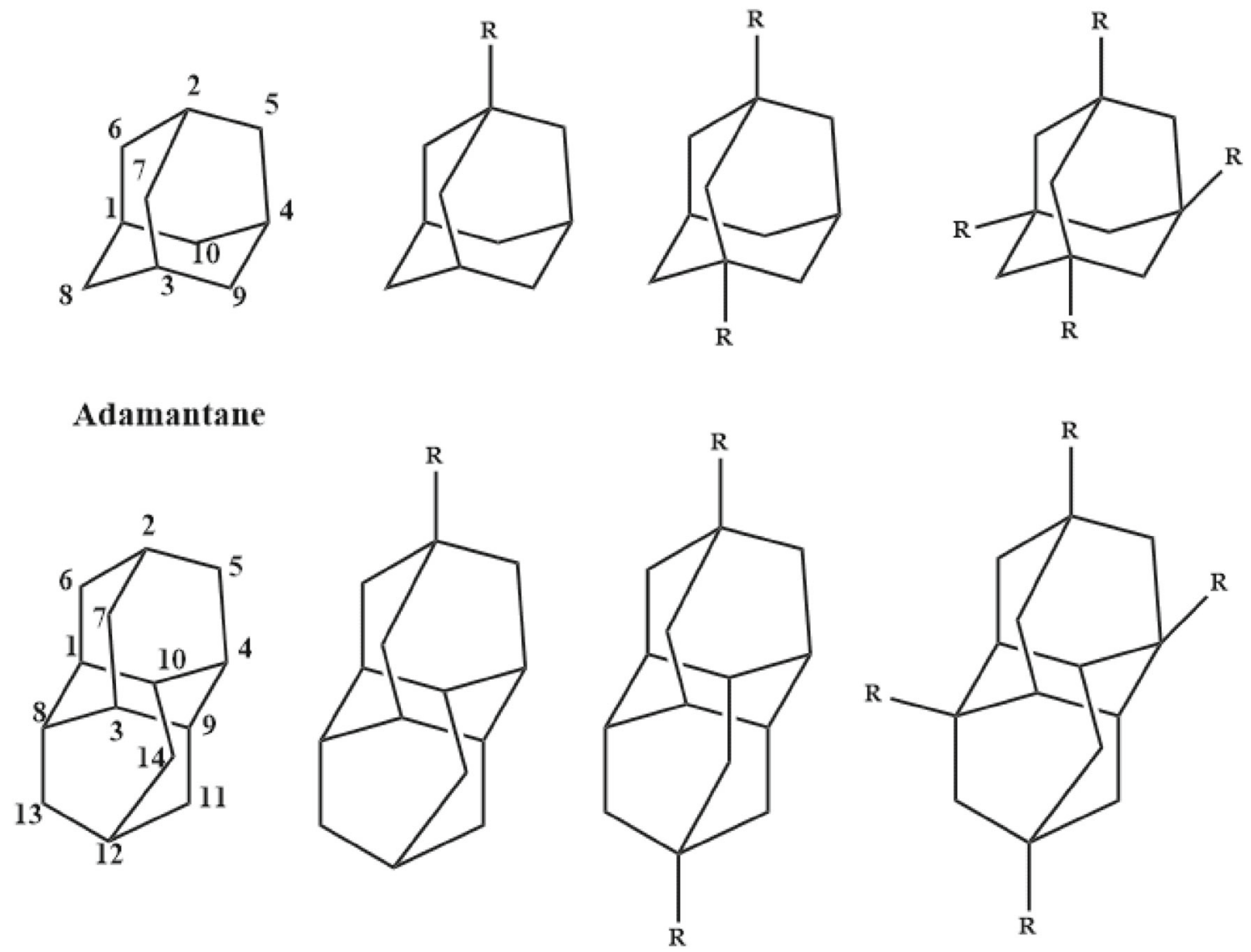

Diamantane

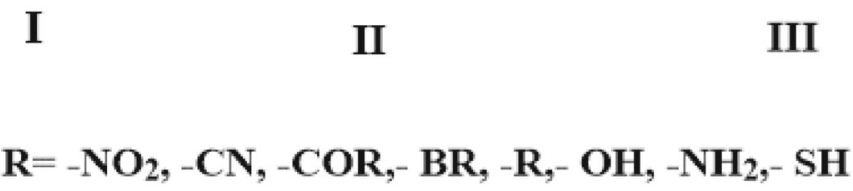

Figure 1. Molecular frameworks of the ADA and DIA molecules and their (homo) functionalized forms obtained by the push and pull-type groups. The labels I, II and III indicate the mono, di, and tetra-substitutions at the tertiary positions.

$\mathrm{IP}=E_{\text {cation }}-E_{\text {neutral }}$

$\mathrm{EA}=E_{\text {neutral }}-E_{\text {anion }}$

Here, IP refers to the energy required to detach an electron from a neutral molecule whereas the EA refers to the energy released while adding the electron to a neutral molecule. On the other hand, the total energy of the cation(anion) calculated by the removal(addition) of an electron from(to) the optimized neutral species. It is worth mentioning here that the inclusion of zero-point vibrational energy to the total energies yields the respective adiabatic quantities.

\section{Results and Discussion}

\subsection{Ground state geometry optimization}

In functionalization studies, the effect of doping and substitution on electronic properties such as the ionization potential, the electron affinity, and the
HOMO-LUMO energy gap is generally examined. In the present work, we focus how such functionalization could also tune the absorption properties. Figure 1 shows the general molecular frameworks of the adamantane and diamantine, hereafter referred as ADA and DIA, respectively. The increasing number of functionalization such as mono-, di-, and tetra- are labelled as I, II and III, respectively. For the purpose, preferably the tertiary sites $(-\mathrm{CH})$ are chosen since it leads to the formation of more stable carbocation compared to the secondary $-\left(\mathrm{CH}_{2}\right)$ positions. On the other hand, the diamondoids possess several sites that are open for the substitutions. However, we choose four tertiary $(1,2,3,4)$ positions in ADA molecule whereas two apical-tertiary $(2,12)$ and two medial-tertiary $(4,8$ or 1,9$)$ in DIA molecule, and the secondary positions are not considered in the present study.

Using the PBE1PBE level of theory with $6-31+G(d, p)$ basis set, the ground electronic state geometries of ADA 
and DIA molecules are optimized, and they (pristine) exhibit $T_{d}$ and $D_{3 d}$ symmetries with the energy values -390.3 and -545.0 a.u., respectively. These numerical values can be used as the reference geometry in order to see the effect of substitutions on the pristine molecules. The variety of functional groups ranging from strong electron withdrawing to strong electron donating are used as an external dopant in the present study, and some of them are listed as $-\mathrm{NO}_{2},-\mathrm{CN}$, - $\mathrm{COR},-\mathrm{Br},-\mathrm{CH}_{3},-\mathrm{OH}$, $-\mathrm{NH}_{2},-\mathrm{SH}$. These groups, at first, affect the geometry of the pristine molecules by means of lowering the symmetry and as a result, it shifts the frontier orbitals. For brevity, we did not show the key geometrical parameters of the small diamondoids and their derivatives here.

\subsection{Electronic and absorption properties}

3.2a Orbital energies and energy gaps: In order to calculate the absorption spectra for the selected diamondoids, the energy levels of the frontier orbitals are first determined. Thus the predicted the energy levels corresponding to HOMO- $n$, HOMO and LUMO, LUMO $+n$ (where $n$ corresponds to the specified level) yield a significant quantity called 'energy gap', which has an intimate relationship with the electronic states. The HOMO-LUMO energy gap obtained for the pure ADA and DIA molecules is 7.91 and $7.38 \mathrm{eV}$, respectively, and these values are tuned by using the different functional groups such as push $\left(-\mathrm{CH}_{3},-\mathrm{OH},-\mathrm{NH}_{2}\right)$ and pull $\left(-\mathrm{NO}_{2},-\mathrm{CN},-\mathrm{COR}\right)$ types. As a result, we achieved the energy gaps in the range of 5.9 to $8.5 \mathrm{eV}$ for the functionalized diamondoids and found the large reduction for the $-\mathrm{COR}$ (where, $\mathrm{R}=\mathrm{Me}$ ) substitution, i.e., the reduction about $1.93 \mathrm{eV}$ and $1.16 \mathrm{eV}$ low in tetra-substituted ADA and DIA molecules, respectively. This may be due to the stabilization of the unoccupied states (LUMOs) which are downshifted; on the other hand, the HOMOs are upshifted. The complete list of frontier orbital energies and energy gaps for the molecules examined in the present study can be found in Tables 1 and 2 . The calculated energy gaps of the pure and functionalized small diamondoids are in close agreement (within the error of our method) with the other theoretical reports. ${ }^{25,26(b), 34}$ There are some reports significantly large reduction in the energy gaps for the carbonyl or nitro group substituent and also by interstitial doping. ${ }^{23-25}$ However, our aim in this study is not to achieve the lowest energy gap but to study the absorption properties. For clarity, we also plotted the orbital energies and energy gaps for the various (homo)functional derivatives of ADA and DIA molecules in Figure 2. It can be seen from Figure 2 that the increasing number of substitutions (such as mono, $d i$, and tetra) in the parent molecule generally decreases

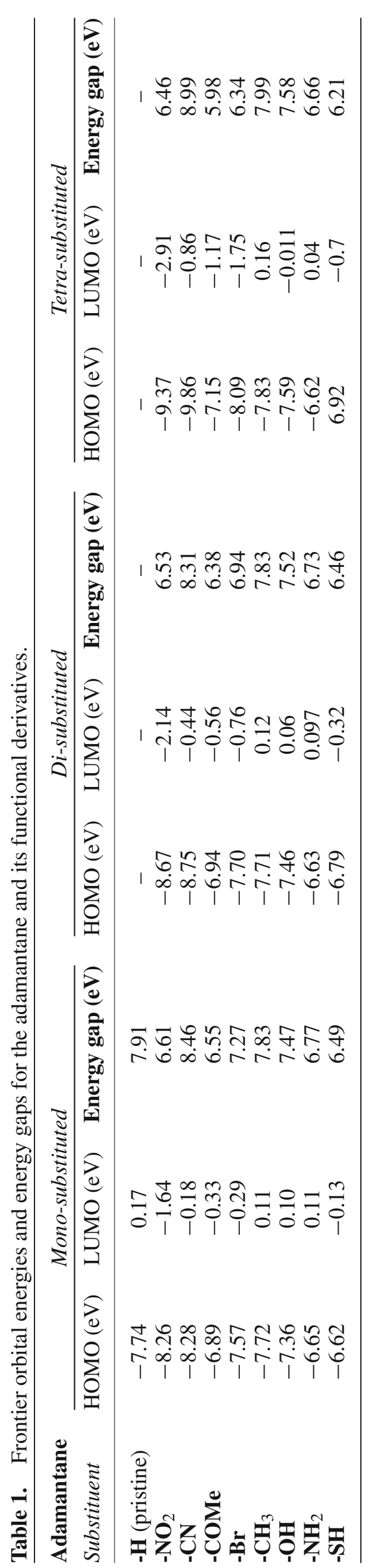




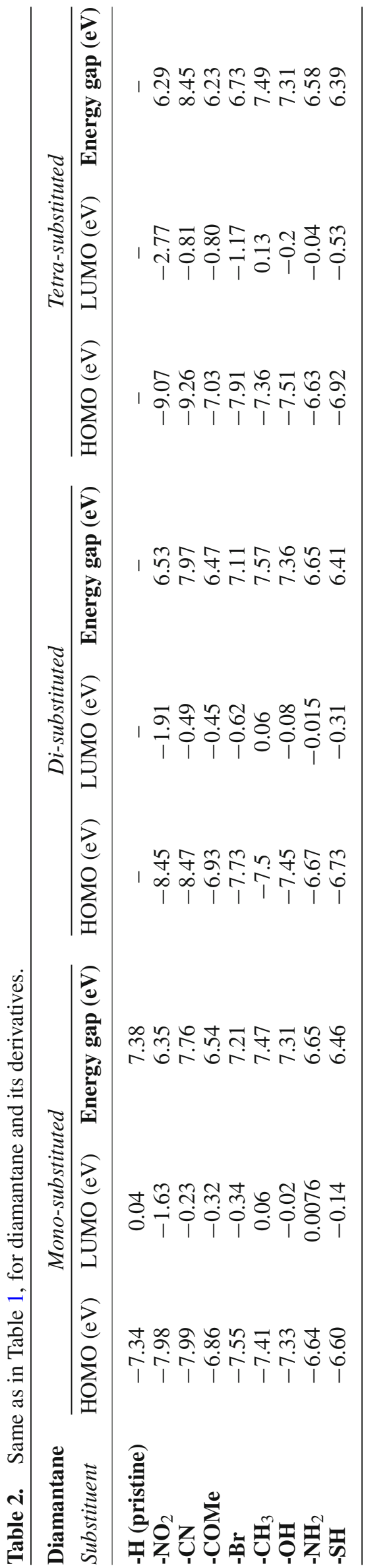

the energy gap, except the cyano group in which the opposite trend is observed.

The frontier orbitals of the pristine and one representative derivative of each type (-OH for push and $-\mathrm{NO}_{2}$ for pull) of smaller diamondoids are depicted in Figures 3-5, respectively. It can be noticed from Figure 3 (pristine) that the HOMO and LUMO are delocalized over the whole molecule. The HOMO is mainly associated with the carbon-carbon bonds, whereas the LUMO shows antibonding character on the carbon-hydrogen bonds. It is reported that the LUMO energies of pristine molecules are positive which indicate that these probably have comparatively high electron affinities. The frontier orbitals of the functionalized ADA and DIA molecules, shown for the $-\mathrm{OH}$ group in Figure 4, clearly illustrate that the LUMOs exhibit high density spread over the carbonhydrogen bonds when compared to that of the HOMOs. The dominant transitions occurred more commonly between HOMO and LUMO for the -OH functionalized smaller diamondoids, whereas such transitions may occur from HOMO- $n$ to LUMO or LUMO $+n$ for the choice of $-\mathrm{NO}_{2}$ group, as can be seen from Figures 5(a) and 5(b). It is also noticed that the LUMOs appear to be high in energy for the former group (push-type) when compared to that of the latter group (pull-type).

3.2b Vertical excitation energies and oscillator strengths: Now, the absorption spectra of the pristine and functionalized ADA and DIA molecules are calculated within the TD-PBE level of theory using 6$31+\mathrm{G}(\mathrm{d}, \mathrm{p})$ basis set. The absorption properties such as vertical excitation energies and the oscillator strengths corresponding to the significant transitions are extracted in the present study. The complete list of dominant orbital contributions of the lowest allowed and most intense transitions observed in the pristine and functionalized ADA and DIA spectra are presented in Table 3 and 4, respectively. For adamantane, the first lowest triply degenerate $\left(T_{2}\right)$ excited states (HOMO-2 $\left(T_{2}\right) \rightarrow$ $\left.\operatorname{LUMO}\left(A_{1}\right)\right)$ at around $6.94 \mathrm{eV}$ account for the lowestenergy absorptions, but a more intense contribution comes from triply degenerate excited states (HOMO$2\left(T_{2}\right) \rightarrow$ LUMO+1( $\left.\left.T_{2}\right)\right)$ at $7.62 \mathrm{eV}$. These can be compared to the experimental spectrum, ${ }^{12}$ where the peaks are observed between $7-8 \mathrm{eV}$ with many sharp features arising from Rydberg states. In the case of diamantane, the lowest transition $\left(\operatorname{HOMO}\left(A_{1 g}\right) \rightarrow\right.$ $\left.\operatorname{LUMO}\left(A_{1 g}\right)\right)$ is symmetry forbidden, in agreement with the findings from Ref. ${ }^{12}$, and it is no good measures for the optical gap. The first available excited state involves a weak $\operatorname{HOMO}\left(A_{1 g}\right) \rightarrow \operatorname{LUMO}+1\left(A_{2 u}\right)$ transition at 

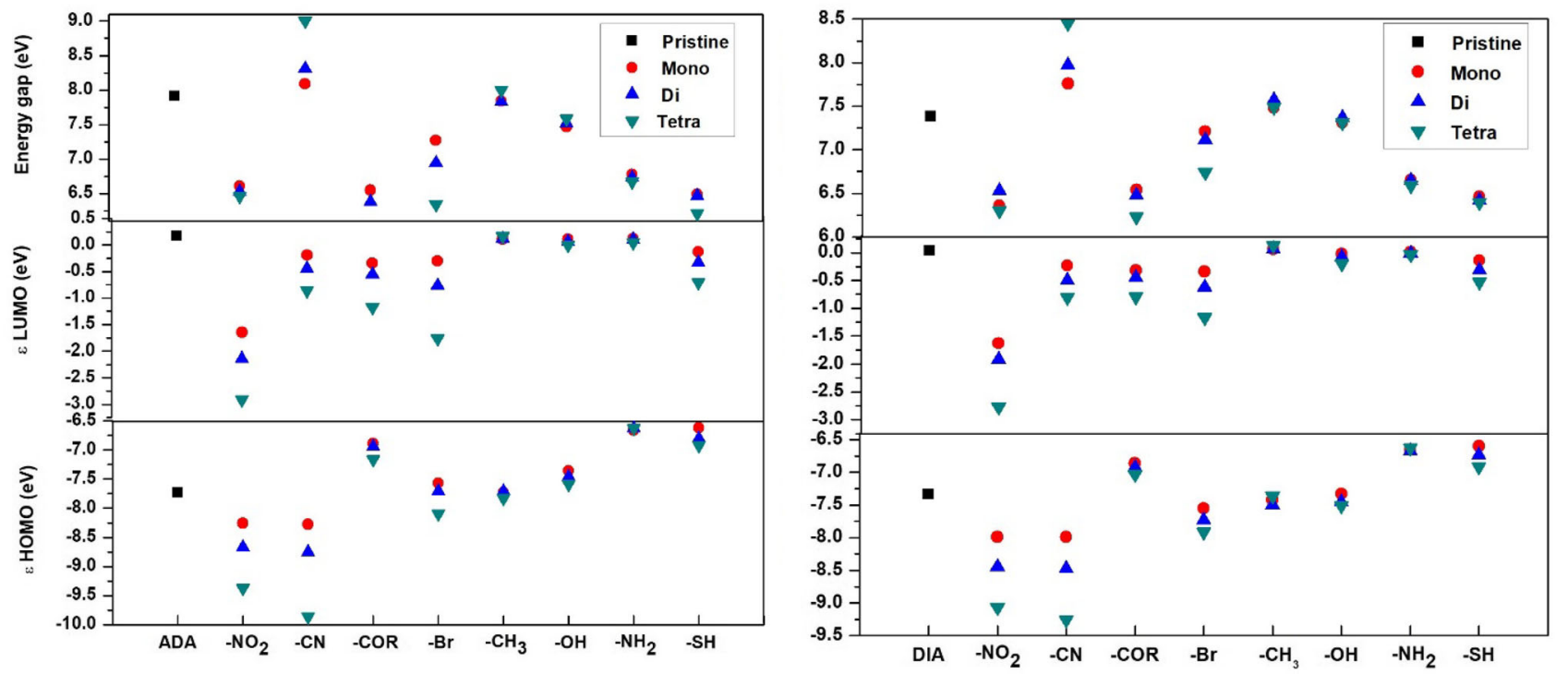

Figure 2. Effect of substituents on the orbital energies and energy gaps of the ADA and DIA molecules. The different line points indicated in the panel for the mono-, di- and tetra-substituted derivatives of the pristine molecule.

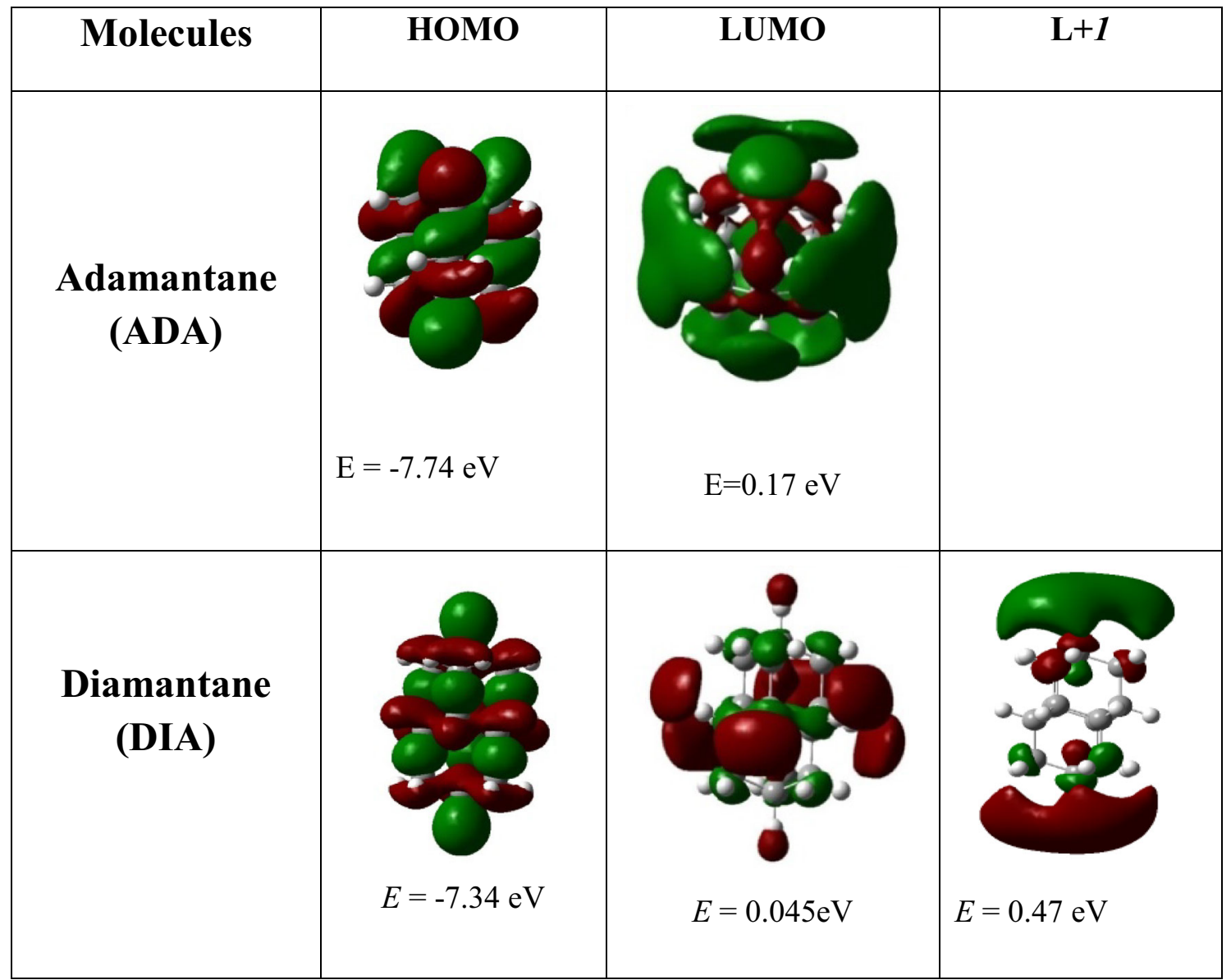

Figure 3. Frontier molecular orbitals of the pristine ADA and DIA molecules. The orbital energies are indicated in the respective panel.

around $6.97 \mathrm{eV}$, and the strongest contributions to the vertical excitation spectrum come from doubly degenerate excited states at $7.28 \mathrm{eV}$ (Table 4).
For clear representation, the vertical excitation energies corresponding to the above said two allowed transitions in the ADA and DIA molecules are also 


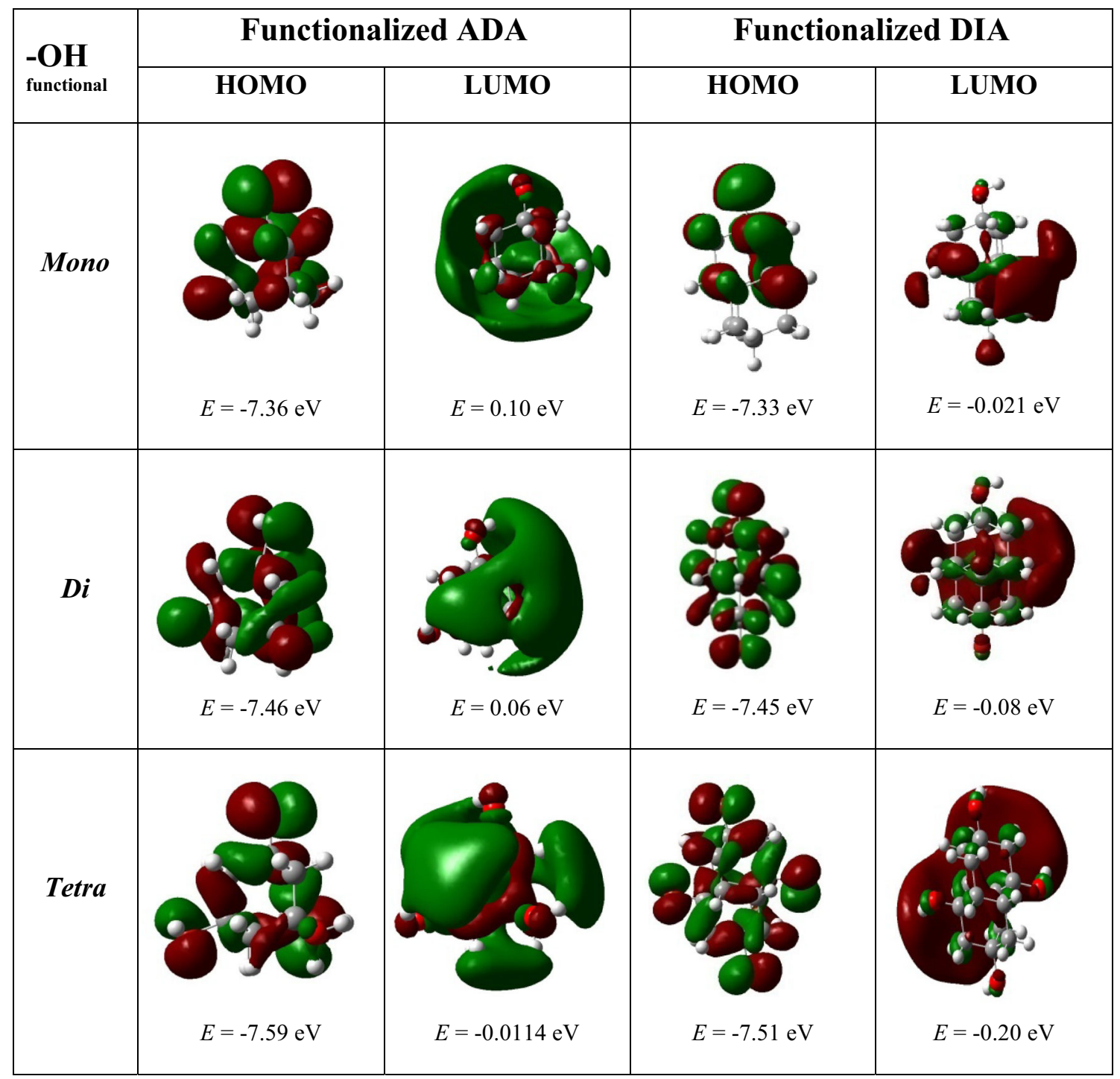

Figure 4. Pictorial representation of the frontier molecular orbitals of the (push-type)-OH functionalized ADA and DIA molecules. Three rows correspond to the increasing order of functionalization mono, di, and tetra, respectively. The orbital energies are included in the respective panel.

presented in the Supplementary Information (SI) as Figure S1-S3. Comparison of these energies for the pristine $(-\mathrm{H})$ diamondoids, Figure S3 clearly reveals the lowest (weak) allowed transition at lower excitation energy and most intense (strong) transition at relatively higher energy. It is worth mentioning here that the low energy transitions are important for the investigations of the radiative and non-radiative decay mechanism such as fluorescence or internal conversion, ${ }^{20}$ and we noticed the significant number of low-energy transitions here for the di- and tetra-substituted diamondoids (Tables 3 and 4). It can also be noticed from Table 3 that the (oscillator) strength of lowest allowed transitions for the pristine ADA molecule is enhanced with the push-type functional groups whereas the same is weakening with the pull-type groups. It is further noticed that the (frontier) highest occupied orbitals involved in the lowest transitions are downshifted (i.e., HOMO-n) with the different choice of pull-type functional groups in ADA molecule. This variation is purely due to the symmetry-distortion (lowering the symmetry from $T_{d}$ ) introduced in the pristine molecule by an externaldoping at the $\mathrm{C}-\mathrm{H}$ site, and the symmetry influences the shape of the frontier orbitals. For illustration, the substitution of one cyano group at tertiary site $\left(2^{\text {nd }}\right.$ position, $c f$. Figure 1) of ADA molecule lowers the symmetry from $T_{d}$ to $C_{3 V}$, and it becomes $C_{2 V}$ and $T_{d}$ $(\mathrm{H}-2 \rightarrow \mathrm{L}+2)$ for the substitution of two (2, 3 positions) and four (1, 2, 3, 4 positions) cyano groups, respectively. There is no systematic variation in the excitation energies of the functionalized smaller diamondoids. On the other hand, they vary randomly for the different 
(a)

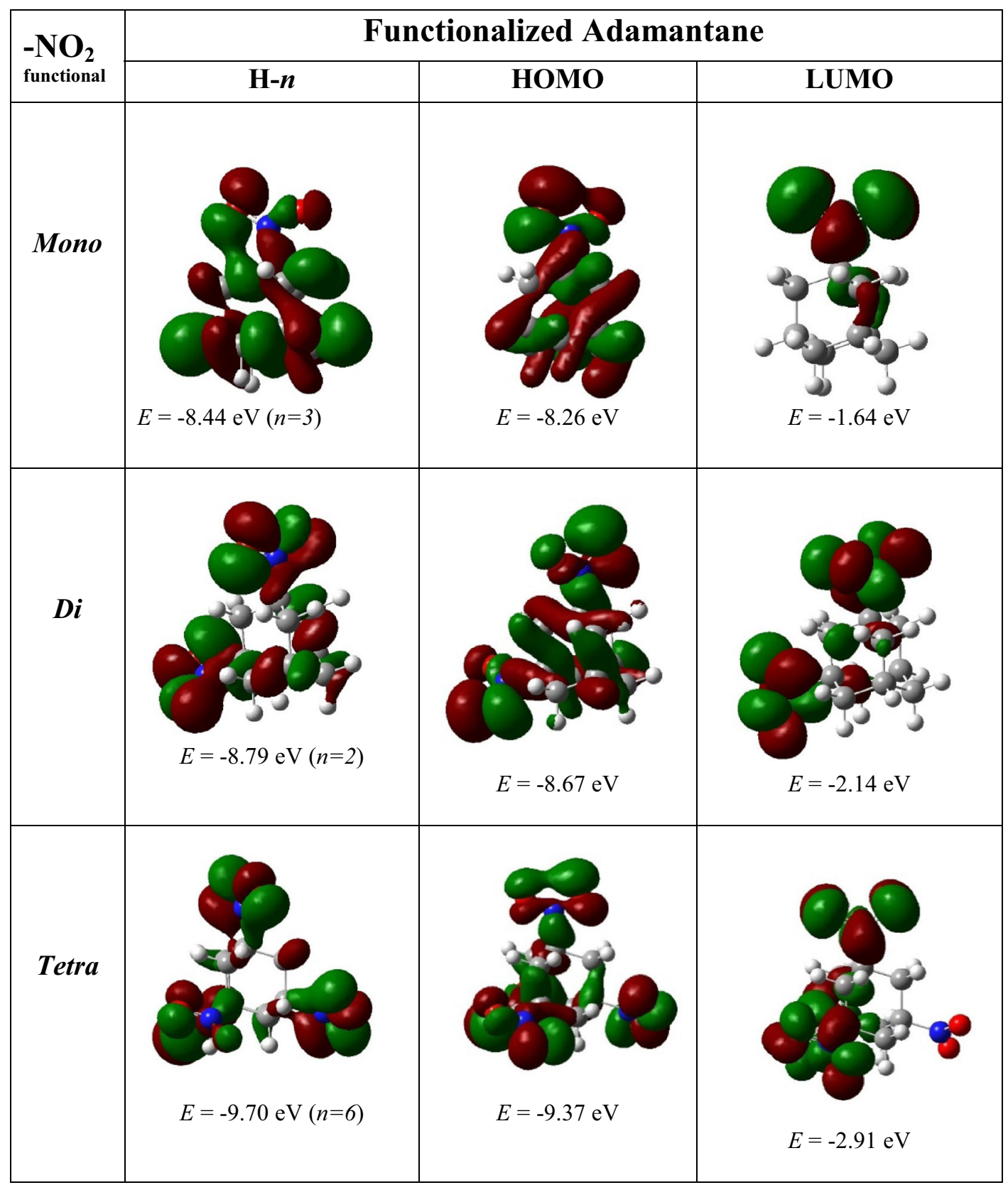

Figure 5. (a) Same as in Figure 4, for the (pull-type) $-\mathrm{NO}_{2}$ functionalized ADA molecule. (b) Same as in Figure 4, for the (pull-type) - $\mathrm{NO}_{2}$ functionalized DIA molecule.

substituents at the tertiary sites. A large reduction in vertical excitation energy about $2.6 \mathrm{eV}$ is found for the lowest weak allowed transition in mono-COR substituted ADA molecules, and the energy is consistently low even for their di- and tetra-substituted molecules. No significant changes in the vertical excitation energy corresponding to the intense transitions.

As observed in the pristine case, the vertical spectra of all the (mono, di and tetra) functionalized ADA molecules show the common trend of a weak allowed transition at lower excitation energy, and an intense transition at relatively higher energy. The functionalized ADA molecules differ in terms of the number of allowed excited states, which reaches the maximum of 25 compared to the pristine, which exhibits only 3 states. Both the weak and intense transition energies are significantly reduced with the increasing number of pull-type functional groups (except $-\mathrm{CN}$ ) whereas the push-type groups slightly increase the transition energies.

Unlike the pristine, the functionalized DIA molecules especially with push-type (-OH, $-\mathrm{NH}_{2}$, and $\left.-\mathrm{SH}\right)$ groups exhibit the HOMO $\rightarrow$ LUMO as the first lowest allowed 
(b)

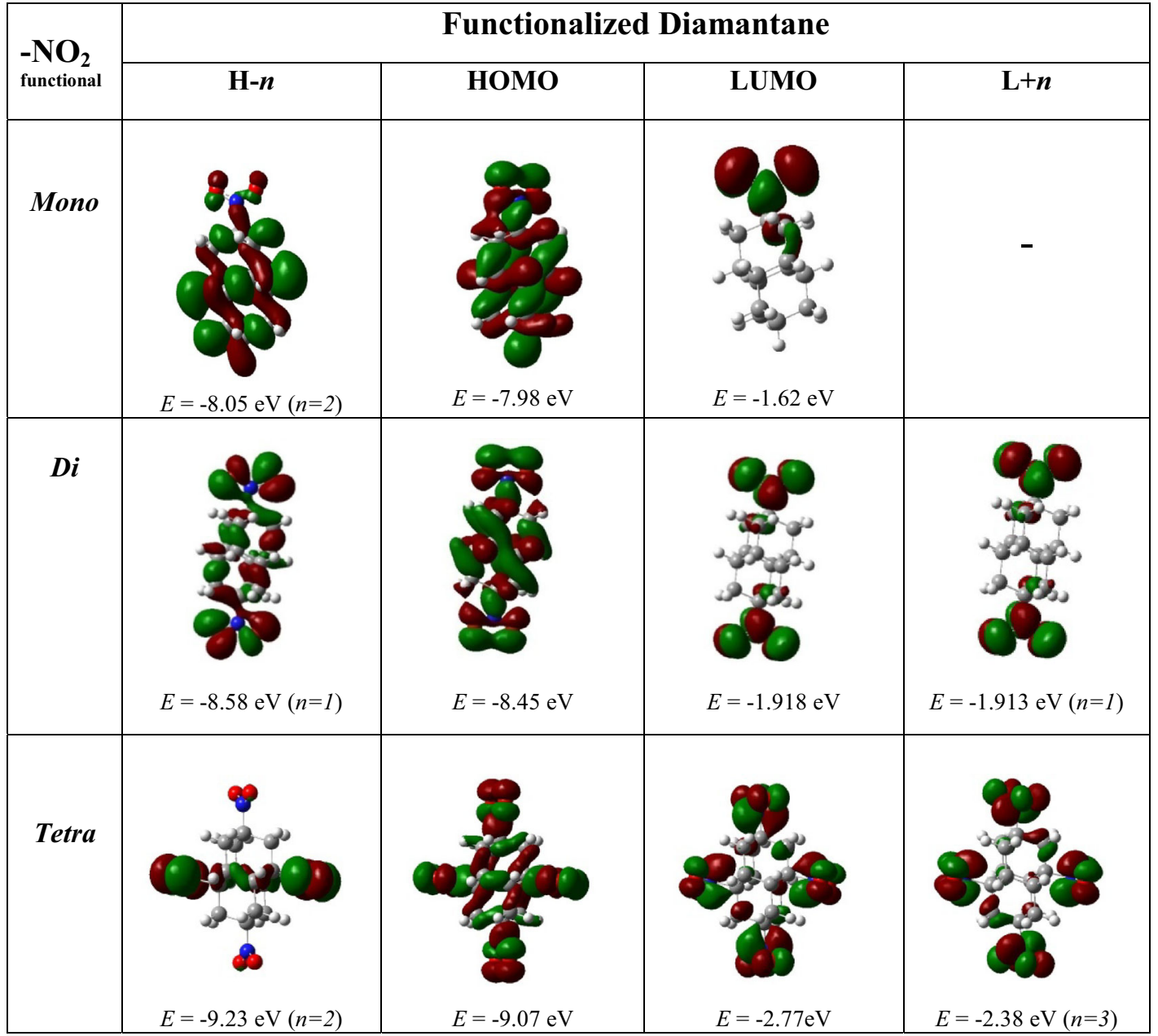

Figure 5. continued

transition with relatively high strength (Table 4). The absorption spectrum of the functionalized DIA molecules, calculated within the TD-PBE level, features the significant peaks that correspond to the lowest and intense allowed transitions at low and high energies, respectively. We observed that the overall trend in the spectrum is same as the functionalized ADA molecules. The significant changes can be seen in the spectrum for the higher diamondoids (i.e., tetramantane, pentamantane), ${ }^{33,34}$ which is beyond the scope of the present study.

\subsection{Ionization Potential and Electron Affinity}

We also calculated the vertical ionization potentials (IP) and affinity energies (EA) by using the PBE1PBE level of theory for the smaller diamondoids, as described in Section 2. It is known that IPs and EAs are closely related to the energies of HOMO and LUMO, respectively, and the data are plotted in Figure 6 for clarity.
Here, the calculated IP value of the pristine ADA molecule (see panel (a) of Figure 6) about $9.42 \mathrm{eV}$ is in relatively good agreement with the experimental IP value $(9.23 \mathrm{eV}),{ }^{27,35}$ and also with the other computational approaches. ${ }^{19,25,26(\mathrm{~d})}$ Upon functionalization, the IP value of ADA molecule is increased with a large number of pull-type substitutions, on the other hand, the external doping by electron-releasing groups decrease the IP value of the pristine ADA molecule. Here, we observed the maximum IP value $(11.29 \mathrm{eV})$ for the tetracyanoadamantane (pull-type) and the lowest value $(8.13 \mathrm{eV})$ for the tetraaminoadamantane (push-type). It can be seen from Figure 6 that the calculated IP value of diamantane is about $0.36 \mathrm{eV}$ lower than that of ADA molecule. It implies that the increasing order of polymantane decreases the IP value which is in good agreement with the experimental prediction of the IPs of polymantanes. ${ }^{35}$ The different functional groups affect the IP of DIA molecule ( $c f$. Figure 6(b)) in the same manner as seen in ADA derivatives. It is noticed that 


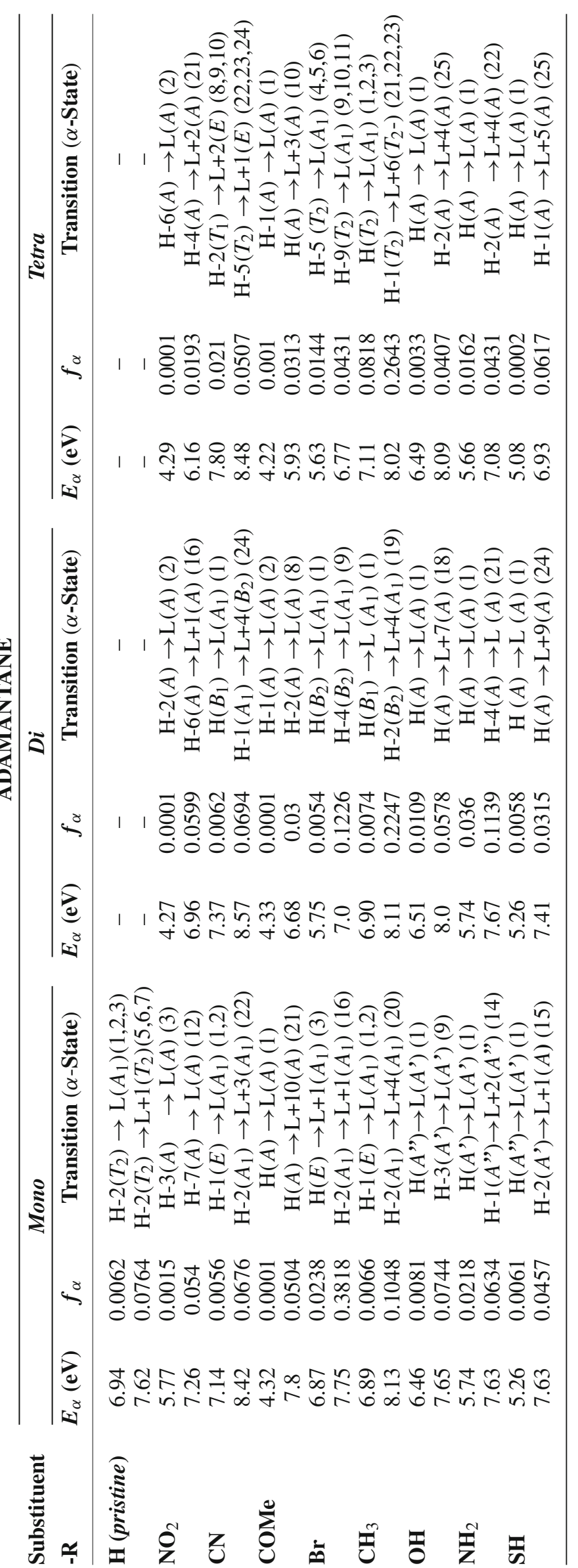




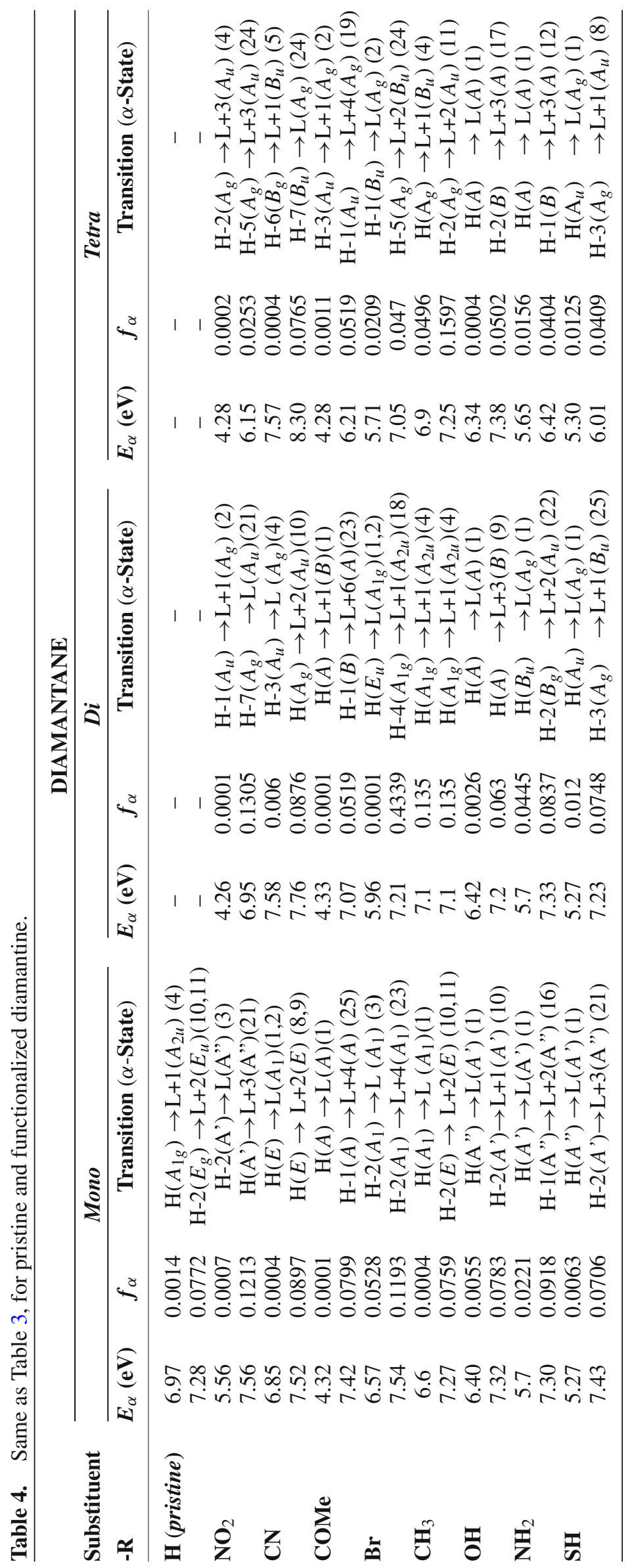



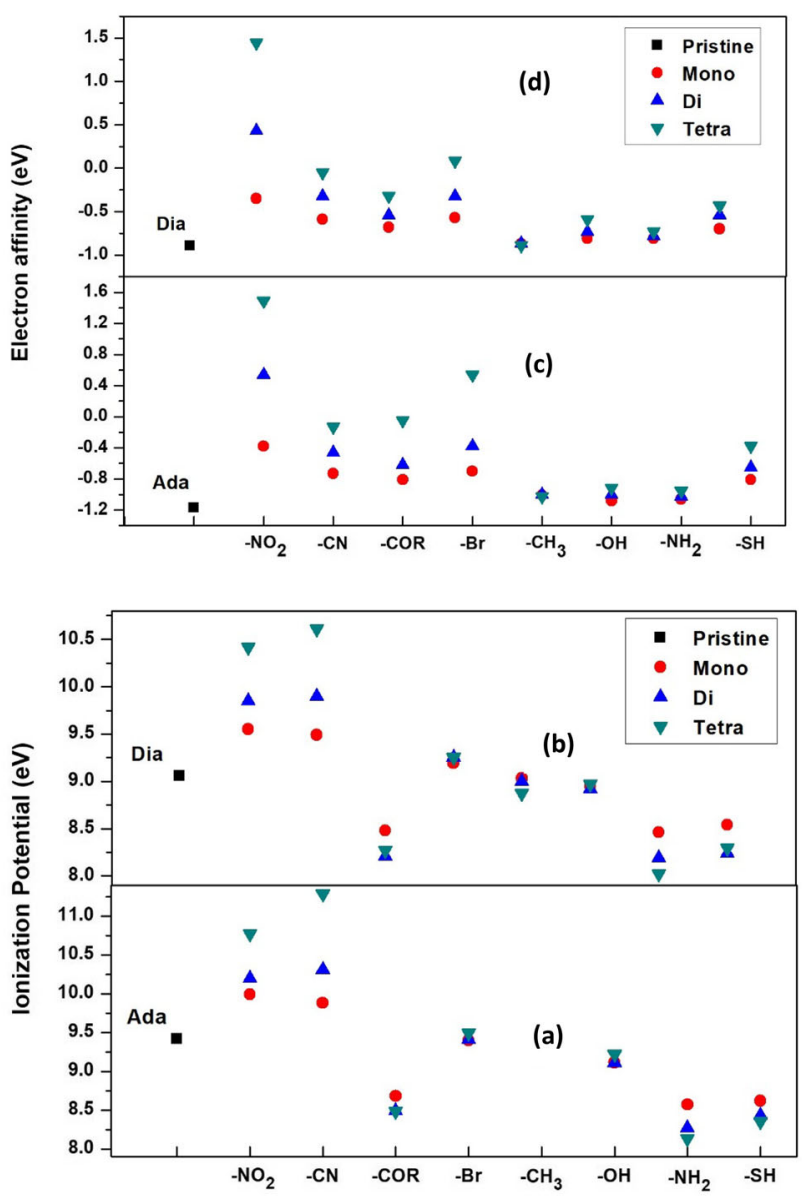

Figure 6. Effect of substituents on the ionization potentials (panels (a)-(b)) and affinity energies (panels (c)-(d)) of the ADA and DIA molecules, respectively. The different line points in the panels indicate the mono-, di-, and tetra-substituted derivatives of each molecule.

the highest IP value $(10.61 \mathrm{eV})$ is for the tetracyano substituted DIA molecule, whereas the lowest IP value $(\sim 8.02 \mathrm{eV})$ is for the tetraaminodiamantane. It is further noticed that all IPs are a positive quantity, which can be inferred as that the neutral species $(\mathrm{X})$ is invariably stable than the cations $\left(\mathrm{X}^{+}\right)$.

Now, the calculated EAs [cf. Eq. (2)] for the pristine ADA and DIA molecules, shown in Figures 6(c) and $6(\mathrm{~d})$ are -1.17 and $-0.89 \mathrm{eV}$, respectively. It can be seen from Figure 6 that the negative EAs of the pristine and functionalized diamondoids indicate that the neutral species is more stable than the corresponding anion $\left(\mathrm{X}^{-}\right)$. On the other hand, the anions are more commonly higher in energy than the neutral species, i.e., $E_{\text {anion }}>$ $E_{\text {neutral }}$, and higher EAs (positive) are associated with the more stable anions, i.e., $E_{\text {neutral }}>E_{\text {anion }}$. The negative EA opens up to the possibility of coating surfaces with diamondoids to produce new electron-emission devices. In particular, thiolated diamondoids used in a single-molecule conduction junctions and these may act as switches, gates, or transport elements. ${ }^{5}$ A close look at the EA values indicate that the pristine molecules are more negative than that of the functionalized smaller diamondoids. While the pull-type groups stabilize the anions (i.e., less negative or relatively positive EA), the push-type groups stabilize the neutral species. It is noted that the EA values increase with the increasing number of functional groups, such as mono, di, and tetra $(-0.38$, 0.54 and $1.49 \mathrm{eV}$, respectively). We observed highly positive EA about 1.49(1.44) eV for tetranitro-ADA(DIA) molecule in which anionic form is found to be more stable. The effect on the EAs of the pristine diamondoids is relatively small, about $0.1-0.3 \mathrm{eV}$ which is too low for the $-\mathrm{Me},-\mathrm{OH},-\mathrm{NH}_{2}$, and - $\mathrm{SH}$ substituents. Overall, the IP value of the considered diamondoid unit is always larger than the respective EA value, which implies that it is easier to add an electron to a diamondoid than to remove an electron from it.

\section{Conclusions}

We studied the electronic and absorption properties of the pristine and functionalized smaller diamondoids (ADA and DIA) by the DFT and TD-DFT approaches within the PBE1PBE level of theory using the 6$31+\mathrm{G}(\mathrm{d}, \mathrm{p})$ basis set. For the purpose of functionalization, we have chosen four tertiary (-C-H site) positions of the diamondoids and the different types of functional groups, listed as $-\mathrm{NO}_{2},-\mathrm{CN},-\mathrm{COR},-\mathrm{Br},-\mathrm{CH}_{3},-\mathrm{OH}$, $-\mathrm{NH}_{2},-\mathrm{SH}$ in the present study. At first, the substitution of above-said groups stabilizes the frontier orbitals and therefore the electronic and absorption properties are affected. The largest reduction was found in the HOMOLUMO energy gap for the tetra-acylated ADA and DIA molecules, and the gap is reported to be 5.98 and $6.22 \mathrm{eV}$, respectively. The frontier orbital energy diagrams for the pristine and functionalized smaller diamondoids are presented. The stability of the functionalized diamondoids is also studied by calculating the IP and EA values. It is found that all IPs are positive quantities for the pristine and functionalized diamondoids, and it implies that the monovalent cations are highly unstable (about $11.29 \mathrm{eV}$ too high) with respect to their neutral species. The negative quantity of EA of the pristine molecules infers again that the neutral is relatively stable than anions. While the anions of the functionalized smaller diamondoids are strongly stabilized by the pull-type groups, the push-type groups slightly stabilize them.

With the advent of time-dependent DFT approach, the absorption bands and excitations were explicitly calculated by considering 25 states. The lowest triply degenerate excited states correspond to the $\mathrm{HOMO} \rightarrow$ LUMO 
transition for the pristine ADA molecule, whereas the DIA molecule shows the weak HOMO $\rightarrow$ LUMO+1 transition for the first available (non-degenerate) excited state. The vertical excitation energies of the pristine diamondoids are significantly reduced by the pull-type functional groups ( $c f$. Tables 3 and 4, for tetranitro and tetraacyl derivatives) compared to the push-type groups. Overall, we made an attempt to contribute towards better insights into the electronic and absorption properties of the smaller diamondoids due to the substitution of different functional groups. The functionalized diamondoids show some potential applications in the medicinal chemistry and organic electronics (electron-emission devices) and we believe that this study might give some insights into the chemistry of diamondoids.

\section{Supplementary Information (SI)}

The energy values for the pristine and functionalized diamondoids are presented as Table S1 (for ADA) and Table S2 (for DIA molecule) in the SI. The calculated absorption spectra are also available as figures in the SI. Supplementary Information is available at www.ias.ac.in/chemsci.

\section{Acknowledgements}

This study is partially supported by the University Start-up grant (No. PU/PC/Start-up-Grant/2011-12/575), Pondicherry University, India. One of the authors (MJKK) also thanks Pondicherry University for the $\mathrm{PhD}$ research fellowship.

\section{References}

1. Wang Y, Kioupakis E, Lu X, Wegner D, Yamachika R, Dahl J E, Carlson R M K, Louie S G and Crommie M F 2008 Spatially resolved electronic and vibronic properties of single diamondoid molecules Nat. Mater. 738

2. Schnell J R and Chou J 2008 Structure and mechanism of the M2 proton channel of influenza A virus Nature 451591

3. Zhang G 2013 Diamondoid molecules: With applications in biomedicine, materials science, nanotechnology and petroleum science Phys. Today $\mathbf{6 6} 59$

4. (a) Mansoori G A, De Araujo P L B and De Araujo E S 2012 Diamondoid molecules: With applications in biomedicine, material science, nanotechnology and petroleum science (World Scientific, Hackensack, USA); (b) Mansoori G A 2013 Diamondoids-the molecular lego of biomedicine, materials science and nanotechnology J. Bioanal. Biomed. 5116

5. Schwertfeger H, Fokin A A and Schreiner P R 2008 Diamonds are a chemist's best friend: Diamondoid chemistry beyond adamantane Angew. Chem. Int. Ed. 471022

6. Yang W L, Fabbri J D, Willey T M, Lee J R I, Dahl J E, Carlson R M K, Schreiner P R, Fokin A A, Tkachenko B A, Fokina N A, Meevasana W, Mannella N, Tanaka K, Zhou X J, Buuren T van, Kelly M A, Hussain Z, Melosh
N A and Shen Z-X 2007 Monochromatic electron photoemission from diamondoid monolayers Science 3161460

7. (a) Landa S and Machacek V 1933 Sur l'adamantane, nouvel hydrocarbure extrait du naphte Coll. Czech. Chem. Comm. 5 1; (b) Prelog V and Seiwerth R 1941 Über die Synthese des Adamantans Ber. Dtsch. Chem. Ges. A/B74 1644

8. Schleyer P von R 1957 J. Am. Chem. Soc. 79 3292

9. (a) Cupas C A, Schleyer P von R and Trecker D J 1965 Congressane J. Am. Chem. Soc. 87 917; (b) Williams V Z, von P, Schleyer R, Gleicher G J and Rodewald L B 1966 Triamantane J. Am. Chem. Soc. 88 3862; (c) Burns W, Mitchell T, McKervey M, Rooney J, Ferguson G and Roberts P 1976 Gas-phase reactions on platinum: Synthesis and crystal structure of anti-tetramantane, a large diamondoid fragment J. Chem. Soc. Chem. Commun. 21 893; (d) Schleyer P von R 1990 My Thirty Years in Hydrocarbon Cages: From Adamantane to Dodecahedrane In Cage Hydrocarbons G A Olah (Ed.) (New York: John Wiley) p. 1

10. Lin R and Wilk Z A 1995 Natural occurrence of tetramantane $\left(\mathrm{C}_{22} \mathrm{H}_{28}\right)$, pentamantane $\left(\mathrm{C}_{26} \mathrm{H}_{32}\right)$ and hexamantane $\left(\mathrm{C}_{30} \mathrm{H}_{36}\right)$ in a deep petroleum reservoir Fuel $\mathbf{7 4}$ 1512

11. Dahl J E, Liu S and Carlson R M K 2003 Isolation and structure of higher diamandoids, nanometer-sized diamandoid molecules Science 29996

12. Landt L, Klünder K, Dahl J E, Carlson R M K, Möller $\mathrm{T}$ and Bostedt C 2009 Optical response of diamond nanocrystals as a function of particle size, shape, and symmetry Phys. Rev. Lett. 103047402

13. (a) Meador M A 1998 Recent advances in the development of processable high-temperature polymers Annu. Rev. Mater. Sci. 28 599; (b) G Ali Mansoori, Thomas F George, Lahsen Assoufid and Guoping Zhang (Eds.) 2007 Molecular Building Blocks for Nanotechnology (New York: Springer); (c) Mansoori G A 2007 Diamondoid molecules Adv. Chem. Phys. 136 207

14. (a) Huang H, Pierstorff E, Osawa E and Ho D 2007 Active nanodiamond hydrogels for chemotherapeutic delivery Nano. Lett. 7 3305; (b) Stouffer A L et al. 2008 Structural basis for the function and inhibition of an influenza virus proton channel Nature 451 596; (c) Geldenhuys W J, Malan S F, Bloomquist J R, Marchand A P and Schyf C J V 2005 Pharmacology and structure-activity relationships of bioactive polycyclic cage compounds: A focus on pentacycloundecane derivatives Med. Res. Rev. 25 21; (d) Lukas W, Khalid I and Schreiner P R 2013 Chem. Rev. 1133516

15. Gunawan M A, Hierso J C, Poinsot D, Fokin A A, Fokina N A, Tkachenko B A and Schreiner P R 2014 Diamondoids: Functionalization and subsequent applications of perfectly defined molecular cage hydrocarbons New $\mathrm{J}$. Chem. $\mathbf{3 8} 28$

16. Chan Y C, Choy K, Chan A H C, Ng K M, Liu Shenggao, Sciamanna S F, Dahl J E P and Carlson R M K 2008 Solubility of diamantane, trimantane, tetramantane, and their derivatives in organic solvents J. Chem. Eng. Data $\mathbf{5 3} 1767$ 
17. (a) Vörös M, Demján T, Szilvási T and Gali 2012 A Tuning the Optical gap of nanometer-size diamond cages by sulfurization: A time-dependent density functional study Phys. Rev. Lett. 108 267401; (b) Demján T, Vörös M, Palummo M and Gali A 2014 Electronic and optical properties of pure and modified diamondoids studied by many-body perturbation theory and time-dependent density functional theory J. Chem. Phys. 141064308 and references therein

18. McIntosh G C, Yoon M, Berber S and Tomanek D 2004 Diamond fragments as building blocks of functional nanostructures Phys. Rev. B 70045401

19. Marsusi F, Mirabbaszadeh K and Mansoori G A 2009 Opto-electronic properties of adamantane and hydrogenterminated sila-and germa-adamantane: A comparative study Physica E 411151

20. (a) Landt L, Staiger M, Wolter D, Klünder K, Zimmermann P, Willey T M, van Buuren T, Brehmer D, Schreiner P R, Tkachenko B A, Fokin A A, Möller T and Bostedt C 2010 The influence of a single thiol group on the electronic and optical properties of the smallest diamondoid adamantane J. Chem. Phys. 132 024710; (b) Landt L, Bostedt C, Wolter D, Möller T, Dahl J E P, Carlson R M K, Tkachenko B A, Fokin A A, Schreiner P R, Kulesza A, Mitrić R and Bonačić-Koutecký V 2010 Experimental and theoretical study of the absorption properties of thiolated diamondoids J. Chem. Phys. 132144305

21. Lu A J, Pan B C and Han J G 2005 Electronic and vibrational properties of diamond like hydrocarbons Phys. Rev. B 72035447

22. Garcia J C, Justo J F, Machado W V M and Assali L V C 2009 Functionalized adamantane: Building blocks for nanostructure self-assembly Phys. Rev. B 80125421

23. Fokin A A and Schreiner P R 2009 Band gap tuning in nanodiamonds: First principle computational studies Mol. Phys. 107823

24. Rander T, Staiger M, Richter R, Zimmermann T, Landt L, Wolter D, Dahl J E, Carlson R M K, Tkachenko B A, Fokina N A, Schreiner P R, Möller T and Bostedt C 2013 Electronic structure tuning of diamondoids through functionalization J. Chem. Phys. 138024310

25. Teunissen J L, Proft F D and De Vleeschouwer F 2017 Tuning the HOMO-LUMO energy gap of small diamondoids using inverse molecular design J. Chem. Theory Comput. 131351

26. (a) Fyta M 2014 Stable boron nitride diamondoids as nanoscale materials Nanotechnology 25 365601; (b) Natterer A, Adhikari B and Fyta M 2016 Complexes of carbene-functionalized diamondoids and metal atoms: Electronic properties J. Organomet. Chem. 815
8; (c) Adhikari B, Sivaraman G and Fyta M 2016 Diamondoid-based molecular junctions: A computational study Nanotechnology 27 485207; (d) Sarap C S, Adhikari B, Meng S, Uhlig F and Fyta M 2018 Optical properties of single- and double-functionalized small diamondoids J. Phys. Chem. A 1223583

27. Rander T, Bischoff T, Knecht A, Wolter D, Richter R, Merli A and Möller T 2017 Electronic and optical properties of methylated adamantanes J. Am. Chem. Soc. 139 11132

28. Abdulsattar M A, Abduljalil H M and Al-Aaraji N A T 2016 Spectroscopic properties of AlSb nanocrystals using diamondoid structures: A density functional theory study Nanomater. Nanotecnol. 619 and references therein

29. Frisch M J, Trucks G W and Schlegal H B et al 2010 Gaussian 09, Rev. B. 01 (Wallingford, CT: Gaussian, Inc.)

30. (a) Perdew J P, Burke K and Ernzerhof M 1996 Generalized gradient approximation made simple Phys. Rev. Lett. 77 3865; Erratum: ibid., 1997 Generalized gradient approximation made simple 78 1396; (b) Adamo C and Barone V 1999 Toward reliable density functional methods without adjustable parameters: The PBE0 model $J$. Chem. Phys. 1106158

31. (a) Adamo C and Jacquemin D 2013 The calculations of excited-state properties with time-dependent density functional theory Chem. Soc. Rev. 42 845; (b) Laurent A D, Adamo C and Jacquemin D 2014 Dye chemistry with time-dependent density functional theory Phys. Chem. Chem. Phys. 1614334

32. Dennington R, Keith $\mathrm{T}$ and Millam J et al 2009 In Gauss View, Version 5 (Shawnee Mission, KS: Semichem Inc.)

33. Vörös M and Gali A 2009 Optical absorption of diamond nanocrystals from ab initio density-functional calculations Phys. Rev. B 80161411

34. (a) Banerjee S and Saalfrank P 2014 Vibrationally resolved absorption, emission and resonance Raman spectra of diamandoids: A study based on timedependent correlation functions Phys. Chem. Chem. Phys. 16 144; (b) Banerjee S, Stuker T and Saalfrank P 2015 Vibrationally resolved optical spectra of modified diamondoids obtained from time-dependent correlation function methods Phys. Chem. Chem. Phys. 1719656

35. Lenzke K, Landt L, Hoener M, Thomas H, Dahl J E, Liu S G, Carlson R M K, Möller T and Bostedt C 2007 Experimental determination of the ionization potentials of the first five members of the nanodiamond series $J$. Chem. Phys. 127084320 